\title{
Enfermedad de McArdle: descripción de cuatro hermanos con déficit de miofosforilasa
}

\author{
A. LÓPEZ MARTÍN*, R.I. BAÑOS MADRID*, J. GARCÍA-ESTAÑ CANDELA, \\ B. GARCÍA PÉREZ, F.J. PÉREZ BAUTISTA, P. SALMERÓN
}

Servicio de Medicina Interna. *Servicio de Medicina del Aparato Digestivo. Hospital Universitario Virgen de la Arrixaca. Murcia

\author{
MCARDLE DISEASE: REPORT OF FOUR BROTHERS \\ WITH MYOPHOSPHORYLASE DEFICIENCY
}

\begin{abstract}
RESUMEN
La deficiencia de miofosforilasa o enfermedad de McArdle es una entidad rara. El gen para la enzima miofosforilasa ha sido clonado y localizado en el cromosoma 11. La enfermedad se hereda de forma autosómica recesiva con un predominio en los varones. El diagnóstico se establece por la elevación en el contenido de glucógeno y la reducción de la actividad de la enzima en la biopsia de tejido muscular. Presentamos cuatro hermanos de 16,15,11 y 5 años con enfermedad de Mc Ardle. Consultaron por dolor, calambres y mioglobinuria tras ejercicio o infección, en uno de los casos, la mioglobinuria causó un fallo renal agudo. Tres de los casos demostraron una reducción de la actividad de la fosforilasa en la biopsia muscular. Nosotros comentamos los aspectos diagnósticos y también las diferentes posibilidades terapéuticas.
\end{abstract}

PALABRAS CLAVE: Enfermedad de McArdle. Deficiencia de miofosforilasa tipo $\mathrm{V}$.

\begin{abstract}
Myophosphorylase deficiency, or McArdle disease, is an uncommon entity. The gene for human myophosphorylase has been cloned and is located on chromosome 11, in keeping with the autosomal recessive nature of the disease and there is an excess of male patients. The diagno sis is established by documentation of elevated glycogen content and reduced phosphorylase activity in biopsied muscle tissue. We report four cases with McArdle disease which were 16,15,11 and 5 years old. They were brothers, and they came to the hospital because of pain, cramps and myoglobinuria after exercise or infection; in the first case, a male patient, myoglobinuria caused acute renal failure. Three of them showed reduced phosphorylase activity in biopsied muscle tissue. We discuss the different therapeutic possibilities.
\end{abstract}

KEY WORDS: McArdle disease. Myophosphorylase deficiency type $V$.

López Martín A, Baños Madrid RI, García-Estañ Candela J, García Pérez B, Pérez Bautista FJ, Salmerón P. Enfermedad de McArdle: descripción de cuatro hermanos con déficit de miofosforilasa. An Med Interna (Madrid) 2001; 18: 136-138.

\section{INTRODUCCIÓN}

Esta enfermedad fue descrita por Mc Ardle en 1951 (1). Posteriormente, Schmidt y Mahler (2) demostraron que los sujetos que la padecían presentaban una ausencia de la enzima miofosforilasa en su músculo esquelético. Se han identificado hasta 9 alteraciones enzimático-metabólicas en las vías de degradación del glucógeno y de la glucolisis anaerobia. En concreto, la tipo V o enfermedad de Mc Ardle se caracteriza por un déficit de miofosforilasa, por lo que se bloquea la degradación del glucógeno muscular a glucosa-1-fosfato. El rasgo clínico más característico es la limitación para desarrollar ejercicio físico por la aparición de calambres musculares. Se observa un predominio en los varones, en los que además se describe una mayor incidencia de fracaso renal agudo por mioglobinuria. Su incidencia es muy baja; prueba de esto serían los 130 casos descritos en toda la literatura. Por este motivo, a continuación describimos los casos de cuatro hermanos que ingresaron por cuadros de dolores musculares y debilidad tras esfuerzo físico o infección; uno de ellos presentó un fracaso renal agudo, hecho poco frecuente en el curso de la enfermedad; sólo se han observado 16 casos a nivel mundial.

\section{CASOS APORTADOS}

Caso 1: Varón de 16 años que consulta por calambres, debilidad en las extremidades y coluria. Sin antecedentes de interés. En los días previos padeció una faringoamigdalitis con fiebre alta por la que reci-

Trabajo aceptado: 9 de Julio de 1999

Correspondencia: Aurelio López Martín. Servicio de Aparato Digestivo. Hospital Universitario. Virgen de la Arrixaca. Ctra. Cartagena-Murcia s/n. 30120 El Palmar (Murcia). 
bió tratamiento con antibióticos. Cuando ingresó, el enfermo estaba febril y tenía la faringe enrojecida, con placas. Exploración neurológica: fuerza muscular grado III/ $\mathrm{V}$ en las extremidades superiores, no vence la resistencia en extremidades superiores, en las inferiores presenta grado III/V en la musculatura proximal; resto de la exploración normal. En los análisis destaca una CPK superior a 150.000 U/1, creatinina $2 \mathrm{mg} / \mathrm{dl}$, f. alcalina $179 \mathrm{U} / \mathrm{l}$, GPT $1287 \mathrm{U} / \mathrm{l}$, GOT $184 \mathrm{U} / \mathrm{l}$, aldolasa 6,3 U/l, LDH $1800 \mathrm{U} / \mathrm{l}$, VSG $30 \mathrm{~mm}$ la primera hora, mioglobinuria positiva. Se realizó el test de isquemia del antebrazo y se observó en él una curva plana para el ácido láctico y un aumento del amonio. El estudio electromiográfico mostró cambios compatibles con miopatía. En la biopsia muscular, y tras realizar la técnica de inmunohistoquímica, se confirmó la ausencia de actividad de la enzima miofosforilasa. Al tercer día del ingreso tuvo un fracaso renal agudo con diuresis conservada, con creatinina hasta 4 . La biopsia renal demostró una nefritis túbulo intersticial aguda sin afectación glomerular. A lo largo de su estancia mejoró la función renal, recuperó la fuerza muscular y se normalizó la CPK (180). Por todo ello, establecimos el diagnóstico de enfermedad de McArdle y fracaso renal agudo por mioglobinuria.

Caso 2: Mujer de 15 años que consulta en el Servicio de Urgencias por mialgias intensas en las extremidades inferiores, imposibilidad para la marcha y coluria. Entre sus antecedentes destaca un ingreso dos años antes por un episodio similar pero de menor intensidad y que remitió en una semana y un hermano diagnosticado de enfermedad de McArdle (Caso 1). El día anterior a su ingreso, tras caminar una distancia de 3-4 $\mathrm{km}$, comienza con dolor muscular intenso en extremidades inferiores; posteriormente aparece la coluria. Cuando la exploramos, la enferma está sin fiebre y hemodinamicamente estable pero presenta una imposibilidad de elevar ambas piernas; el resto de la exploración es normal. La evaluación analítica de urgencia muestra una CPK de 230.000, creatinina de 0,6 y mioglobinuria positiva. El EMG es compatible con miopatía. Se realiza la técnica de histoquímica para la detección de la enzima fosforilasa en células musculares, que demuestra una ausencia total de actividad enzimática, por lo que se establece el diagnóstico de glucogenosis tipo $\mathrm{V}$ o enfermedad de Mc Ardle con rabdomiolisis secundaria. Tiene una evolución favorable con recuperación progresiva de la fuerza muscular y normalización de la CPK.

Caso 3: Varón de 11 años, que ingresa para estudio tras el diagnóstico de sus hermanos (casos 1 y 2). En la exploración presenta buen estado general con buen color muco-cutáneo. Exploración cardiopulmonar, abdominal y neurológica normal. El estudio analítico muestra una CPK de 650 con orina normal. El estudio EMG mostró incrementos significativos de polifasia en el músculo explorado con alteraciones en la morfología de los potenciales de unidad motora que podían ser compatibles con miopatía. Con la técnica histoquímica para la detección de la enzima fosforilasa, las células musculares mostraron ausencia total de actividad, por lo que se establece el diagnostico de enfermedad de McArdle.

Caso 4: Varón de 5 años, hermano menor de los anteriores, que presenta tras una excursión en bicicleta un cuadro agudo de dolores musculares en los miembros inferiores con debilidad intensa sin coluria. Su padre, informado adecuadamente de la enfermedad de sus hijos mayores, decide no acudir a ningún centro hospitalario y lo trata con abundantes líquidos, reposo, dieta rica y variada, con lo que mejora progresivamente en los días posteriores. No se realizó estudio.

\section{DISCUSIÓN}

La enfermedad de Mc Ardle es hereditaria, de forma autosómica recesiva, ligada a la alteración de material genético perteneciente al cromosoma 11. El gen que controla la síntesis de la enzima miofosforilasa se ha podido clonar y determinar su secuencia dentro del cromosoma 11. La consanguinidad de los padres está descrita en muchos casos (3). En esta enfermedad es frecuente encontrar el antecedente de una actividad física importante previa a la aparición de los síntomas. En su evolución, los síntomas pueden aparecer ante esfuerzos pro- gresivamente más pequeños. Hay diferentes formas clínicas de presentación, así podemos encontrar desde una miopatía congénita en la que el paciente no tiene síntoma alguno, hasta una miopatía de curso fatal en la infancia. Una forma intermedia cursa con mioglobinuria y fracaso renal agudo, es más frecuente en varones, lo que podría justificarse por la mayor masa muscular y actividad física que desarrollan (4). El fracaso renal agudo es poco frecuente ya que sólo se han descrito 16 casos en toda la literatura, y tiene buen pronóstico. Esta variabilidad se podría explicar por la ausencia total del enzima en algunos pacientes, mientras que en otros existe en un porcentaje muy inferior al normal (5). El diagnóstico es posible con una clínica compatible y los datos de laboratorio (elevación de la CPK, hasta cifras muy elevadas, que con frecuencia permanecen altas en los periodos asintomáticos de la enfermedad, elevación de la LDH, la aldolasa, y la fosfatasa alcalina, también es posible encontrar una hipogammaglobulinemia (6). Hallazgos electromiográficos compatibles con miopatía. Test de isquemia del antebrazo: se observa una curva plana para el ácido láctico con aumento de amonio hasta diez veces los valores normales, por fallo de la degradación de glucógeno a lactato (7). RMN espectroscópica con $\mathrm{P}^{31}$ : en sujetos sanos se aprecia un incremento en la intensidad de la señal en la musculatura activada con el T 2 que no se aprecia en los enfermos de McArdle (8), esta técnica incruenta es útil para el diagnóstico sobre todo de pacientes asintomáticos (9). Gammagrafía con Tc 99 (hay un aumento de depósito en los grupos musculares afectos). Pero el diagnóstico definitivo se realiza mediante la biopsia muscular, en la que se observa una ausencia de actividad de la enzima miofosforilasa; con técnicas inmunohistoquímicas no se encuentra actividad de la miofosforilasa. La fosforilasa hepática es, generalmente, normal. El rápido progreso en el entendimiento de las bases moleculares de esta enfermedad hará posible el diagnóstico mediante el análisis del ADN leucocitario, como alternativa a la biopsia muscular (10). El método más efectivo para el diagnóstico prenatal de una enfermedad genética con herencia recesiva, una vez identificado el defecto enzimático, es el estudio de los polimorfismos en la región clonada. En la actualidad se podría ofertar a aquellas familias cuyos recién nacidos han fallecido por esta enfermedad. El hallazgo es un polimorfismo tipo Msp-1, inserción -delección en la posición 3 del locus genético, con lo que se obtendría información en el $75 \%$ de los pacientes de alto riesgo (11).

El diagnóstico diferencial debe realizarse con el resto de las miopatías metabólicas, la polimiositis y el déficit de carnitina palmitil transferasa (12).

La prevención y el tratamiento se basan en: a) el control de la actividad física, b) la ingestión previa al ejercicio de glucosa o fructosa, que puede reducir los síntomas, y c) la dieta hiperproteica (13), que puede mejorar la resistencia y funcionalidad muscular, aunque no se ha podido demostrar en la mayoría de los trabajos. En la fase aguda de la rabdomiolisis será aplicará una hidratación enérgica, la alcalinización de la orina y, caso necesario, la hemodiálisis. La taurobetina, un compuesto de taurina, vitamina B6 y B12 podría ser útil. Aunque trabajos como los de Haller y cols., (14) muestran que en esta enfermedad la cantidad de piridoxina presente en el músculo esquelético representa un $20 \%$ de la existente en condiciones normales, su administración no ha tenido éxito; no se obtiene mejoría clínica ni cambios en el estudio anatomopatológico ni aumento de la actividad enzimática tras su empleo (15). 


\section{Bibliografía}

1. McArdle B. Myopathy due to a defect in muscle glycogen breakdown. Cli Sci 1951; 10: 13-36.

2. Schmidt R, Mahler R. Chronic progressive myopathy with myoglobinuria: demonstration of a glycogenolytic defect in the muscle. J Clin invest 1959; 38: 2044-2058.

3. Colomer J, Yoldi M, Torres J. Déficit de fosforilasa muscular en dos hermanos. An Esp Pediatr 1990; 32-2:154-158.

4. De Precigout V, Dupon M, Lacut J, Aparicio M. Insuffisance rénale aiguë au cours d'une glycogénose de Mc Ardle. La Presse Médicale 1989; 18-34: 1709-1710.

5. Feit H, Brooke M. Myophosphorylase deficiency: two different muscular etiologies. Neurology 1976; 26: 963-967.

6. Westarp M, Schreiner V, Kornhuber H. Hypogammaglobulinemia in McArdle myopathy. Eur Arch Psychiatry Clin Neurosci 1991; 240: 159-162.

7. DiMauro S, Bresolin N. Phosphorylase deficiency. IN: Engel Ag. Barker Bu eds. Myology. New York: McGraw-Hill 1986; 1585-1602.

8. Kerviler E, Leroy-Willing A, Jehenson P, Buboc D, Eymard B, Syrota A. Exercise-induced muscle modifications: study of healthy subjects and patients with metabolic myopathies with MR imaging and $\mathrm{P}^{31}$ spectroscopy. Radiology 1991; 181: 259-264.

9. Gruetter R, Kaelin P, Boesch C, Martin E, Werner B. Non-invasive P 31 magnetic resonance spectroscopy revealed McArdle disease in an asymptomatic child. Eur J Pediatr 1990; 149: 483-486.

10. Tsujino S, Shanske S, Dimauro S. Molecular genetic heterogeneity of myophosphorylase deficiency. N Engl J Med 1993; 329: 241-245.

11. Lebo R, Anderson L, DiMauro S, Lynch E, Hwang P, Fletterick R. Rare McArdle disease locus polymorphic site on $11 \mathrm{q} 13$ contains $\mathrm{CpG}$ sequence. Hum Genet 1990; 86: 17-24.

12. Formigo E, Gómez N, Ferreiro JL, Anton L. Retraso en el diagnóstico de la enfermedad de McArdle. An Med Interna (Madrid) 1994; 11: 136-138.

13. Slonim AE, Goans PJ. Myopathy in McArdle's syndrome: improvement with a high-protein diet. N Engl Med 1985; 312: 355-359.

14. Haller RG, Dempsey WB, Feith H, Look JD, Knochel JP. Low muscle levels of piridoxine in McArdle's Syndrome. Amer J Med 1983; 74: 217-220.

15. Grunfeld JP, Ganeval D, Chanard J. Acute renal failure in McArdle's disease. Report of two cases. N Engl J Med 1972; 286: 1237-1241. 